\title{
AN UPPER BOUND TO THE RATE OF IDEAL DISTRIBUTED LOSSY SOURCE CODING OF DENSELY SAMPLED DATA
}

\author{
David L. Neuhoff and S. Sandeep Pradhan \\ EECS Dept., University of Michigan, Ann Arbor, MI
}

\begin{abstract}
Motivated by the question of the efficiency of dense sensor networks for sampling, encoding and reconstructing spatial random fields, this paper uses the Berger-Tung upper bound to the discrete-time distributed rate-distortion function and Grenander-Szego asymptotic eigenvalue theory to obtain an upper bound to the smallest possible rate when using distributed lossy encoding of densely spaced samples that is tighter than the bound recently obtained by Kashyap et al. Both bounds indicate that with ideal distributed lossy coding, dense sensor networks can efficiently sense and convey a field, in contrast to the negative result obtained by Marco et al. for encoders based on time- and space-invariant scalar quantization and ideal Slepian-Wolf distributed lossless coding.
\end{abstract}

\section{INTRODUCTION}

Suppose a spatially distributed random field is sampled by a network of densely spaced sensors, and suppose these samples must be encoded with some loss for transmission to a common decoder, where the field is approximately reconstructed. The question posed in [1,2] (see also [3]) is: What happens to the number of encoded bits per unit area, i.e the encoding rate, as sensor density increases, given a fixed limit on the decoded reconstruction quality? On the one hand, as density increases, more sensor values must be encoded. On the other hand, adjacent values become more correlated, which might be exploited, perhaps, to prevent the encoding rate from growing. A key constraint is that each sensor's encoder must operate without access to the (correlated) outputs of other sensors, since they are not colocated. That is, the encoding must distributed.

To keep things simple, we consider a scenario like that of $[2,4]$, in which a one-dimensional, continuous-space, stationary, Gaussian random process $X(s)$ is sampled at $M$ sites spaced $\tau$ meters apart. Indeed, a temporal sequence $\left(X^{1}(s), X^{2}(s), \ldots\right)$ of independent sample functions from this process is sampled at the given sites. A block of $n$ samples (in time) at each site are block encoded, independently

This work was supported by NSF Grants CCF-0329715 and CCF0448115 (CAREER). of the samples at other sites, for transmission to a common decoder which, after receiving bits from the $M$ sites, outputs continuous-space reproductions $\left(\widehat{X}^{1}(s), \ldots, \widehat{X}^{n}(s)\right)$ for the length $M \tau$ segments of the $n$ continuous-space sample functions. The quality of this reproduction is given by the mean squared error (MSE),

$$
D=\frac{1}{M n \tau} \sum_{i=1}^{n} \int_{0}^{M \tau} E\left(X^{i}(s)-\widehat{X}^{i}(s)\right)^{2} d s .
$$

In $[2,4]$ it was shown that for any $d>0$, if $M=\lfloor 1 / \tau\rfloor$ and if all encoders use a fixed scalar quantizer designed so that when $\tau$ is small the MSE $D$ with linear reconstruction is at most $d$, followed by ideal Slepian-Wolf lossless distributed coding, then the number of bits per unit distance, i.e. the encoding rate or simply rate, tends to infinity as as the sensor density increases, i.e., as $\tau \rightarrow 0$. This negative result contrasts with the fact that if we relax both the distributed encoding and scalar quantization constraints, then it is well known that ideal block coding can attain rate arbitrarily close to the rate-distortion function of the continuousspace process $X$, which is ordinarily finite.

It then becomes important to learn whether the negative result of $[2,4]$ is due to the scalar quantization or to a fundamental limitation of distributed encoding. This was recently answered in [5], which considered the scenario of [2, 4], and found an upper bound to the rate of ideal distributed lossy coding (rather than scalar quantization) applied with distortion at most $d$, which remained bounded as $\tau \rightarrow 0$. One may conclude that it is the scalar quantization, rather than distributed coding per se, that caused the negative result of $[2,4]$.

In this paper, we use the Berger-Tung [6] upper bound to the distributed (discrete-time) rate-distortion function to find a better upper bound to the limiting attainable rate, under the relaxed condition that $M$ is permitted to be large, that is, the sensors are widely distributed as well as dense. The latter permits the use of Grenander-Szego [7] asymptotic eigenvalue theory. Indeed, since recently it was shown in [8] that the Berger-Tung upper bound is tight for the case of discrete-time jointly Gaussian sources with mean squared error criterion, we expect our bound to be tight, as well. 


\section{BACKGROUND}

In the following we briefly review the formulation of distributed source coding of $M$ correlated sources and the BergerTung [6] inner bound on the rate-distortion region, which will lead to an upper bound to the distributed rate-distortion function of interest.

Let $X_{1}, X_{2}, \ldots, X_{M}$ be $M$ discrete memoryless correlated sources ${ }^{1}$ with alphabet $\mathcal{X}_{i}$ for source $X_{i}$ for $i=$ $1,2, \ldots, M$ and with joint distribution $q\left(x_{1}, x_{2}, \ldots, x_{M}\right)$. Let $\hat{\mathcal{X}}_{i}$ denote the reconstruction alphabet associated with source $X_{i}$. Associated with these sources, there exists a tuple of bounded distortion measures $d_{i}: \mathcal{X}_{i} \times \hat{\mathcal{X}}_{i} \rightarrow \mathcal{R}^{+}$ for $i=1,2, \ldots, M$. A distributed source code with parameters $\left(n, \Delta_{1}, \Delta_{2}, \ldots, \Delta_{M}, \Theta_{1}, \Theta_{2}, \ldots, \Theta_{M}\right)$ is characterized by $M$ encoding and decoding functions, respectively, of the form

$$
\begin{gathered}
e_{i}: \mathcal{X}_{i}^{n} \rightarrow\left\{1,2, \ldots, \Theta_{i}\right\} \\
f_{i}:{ }_{j=1}^{M}\left\{1,2, \ldots, \Theta_{j}\right\} \rightarrow \hat{\mathcal{X}}_{i}^{n}
\end{gathered}
$$

which satisfy the following conditions

$$
E d_{i}\left[X_{i}^{n}, f_{i}\left(e_{1}\left(X_{1}^{n}\right), e_{2}\left(X_{2}^{n}\right), \ldots, e_{M}\left(X_{M}^{n}\right)\right)\right] \leq \Delta_{i},
$$

where the distortion between two sequences is obtained as the average of the distortion between pairs of samples.

A tuple of rates and distortions $\left(R_{1}, \ldots, R_{M}, D_{1}, \ldots\right.$, $\left.D_{M}\right)$ is said to be achievable if for all $\epsilon>0$ there exists a distributed source code with parameters $\left(n, \Delta_{1}, \Delta_{2}, \ldots, \Delta_{M}\right.$, $\left.\Theta_{1}, \Theta_{2}, \ldots, \Theta_{M}\right)$ such that for $i=1,2, \ldots, M$, the following hold

$$
\frac{1}{n} \log \Theta_{i} \leq R_{i}+\epsilon, \text { and } \Delta_{i} \leq D_{i}+\epsilon .
$$

The achievable rate-distortion region is the set of all achievable tuples.

Berger-Tung Inner Bound: In [6] an information theoretic characterization of an achievable rate-distortion region is provided. Specifically, a tuple $\left(R_{1}, \ldots, R_{M}, D_{1}, \ldots, D_{M}\right)$ was shown to be achievable if there exists for $i=1,2, \ldots, M$, a conditional distribution $p_{i}(u \mid x)$ defined on some finite alphabet $\mathcal{U}_{i}$ and, also, a tuple of functions $g_{1}, \ldots, g_{M}, g_{i}$ : $\mathcal{U}_{1} \times \mathcal{U}_{2} \ldots \times \mathcal{U}_{M} \rightarrow \hat{\mathcal{X}}_{i}$, such that with

$$
p\left(u_{1}, u_{2}, \ldots, u_{M} \mid x_{1}, x_{2}, \ldots, x_{M}\right)=\prod_{i=1}^{M} p_{i}\left(u_{i} \mid x_{i}\right)
$$

the following hold: (a) $E d_{i}\left(X, \hat{X}_{i}\right) \leq D_{i}, i=1,2, \ldots, M$ and (b) $I\left(X_{S} ; U_{S} \mid U_{S^{c}}\right) \leq R_{S} \quad \forall S \subseteq\{1,2, \ldots, M\}$ where $X_{S}$ is the collection of sources indexed by $S$ (similarly for $\left.U_{S}\right)$ and $R_{S}=\sum_{i \in S} R_{i}$.

\footnotetext{
${ }^{1}$ To simplify notation, the time index is not shown.
}

Note that the random variables $\left\{U_{i}\right\}$ are referred to as auxilliary random variables. This inner bound can be extended to continuous-valued sources with distortion measures which are not bounded using the techniques of [9].

\section{MAIN RESULTS}

For each integer $t$, let $\left\{X^{t}(s):-\infty<s<\infty\right\}$ be a zeromean, unit variance, continuous-space, stationary Gaussian process, denoted $X^{t}$ for short. Subject to a constraint on MSE (defined by (1)), we wish to find an upper bound to the rate (in bits per unit distance) of an ideal distributed lossy source code that operates by encoding spatial samples $X^{t}(1 / N), \ldots, X^{t}(M / N)$ (taken at intervals of $\tau=1 / N$ ) with a distributed code of the kind described in the previous section and then reconstructing an approximate continuousspace sample function. We take the limit first as $M$ becomes large, and then as $N$ becomes large.

We assert that it suffices to focus on the rate and distortion for just the samples. First, the rate in bits per unit distance is simply the average of $R_{1}, \ldots, R_{M}$ (bits per sample) multiplied by the sampling rate $N$ (samples per unit distance). Second, it can be shown that there exists a sequence (indexed by $N$ ) of reconstruction methods such that in the limit the MSE for the continuous-space reproduction equals the average of $D_{1}, \ldots, D_{M}$ for the distributed code. As a result, we can find an upper bound to the least possible rate at a given MSE by finding a particular tuple $\left(R_{1}, \ldots, R_{M}, D_{1}, \ldots, D_{M}\right)$ in the Berger-Tung inner bound region, and then averaging rates and distortions over $M$ and taking limits as $M$ and $N$ increase.

To put the problem in the framework of the previous section, let $Y_{i}$ denote the source consisting of successive samples of $X^{t}(s)$ taken at $s=i / N$. Let $K$ denote the covariance matrix of $Y_{1}, \ldots, Y_{M}$. The achievable tuple in the Berger-Tung inner bound region that we seek is obtained by choosing $p(u \mid x)$ to be that which derives from the following auxiliary random variables:

$$
U_{i}=\frac{1}{1+\phi_{N}}\left(Y_{i}+Z_{i}\right), i=1, \ldots, M
$$

where $\phi_{N} \geq 0$ is an arbitrary number, and $Z_{i}$ is $\mathcal{N}\left(0, \phi_{N}\right)$ and independent of $Y_{i}$. For each $i$, let $\hat{Y}_{i}=g_{i}\left(U_{1}, \ldots, U_{M}\right)$ be the minimum mean squared error (MMSE) estimate of $Y_{i}$ based on $\left\{U_{i}\right\}_{i=1}^{M}$. For this choice, it can be shown that

$$
\frac{1}{M} \sum_{i=1}^{M} E\left(Y_{i}-\hat{Y}_{i}\right)^{2}=\frac{1}{M} \sum_{i=1}^{M} \frac{\lambda_{i}}{\frac{\lambda_{i}}{\phi_{N}}+1},
$$

where $\lambda_{i}$ denotes the $i$ th eigenvalue of $K$. Now let us evaluate the average rate for this choice of distribution. By choosing $R_{i}=I\left(Y_{i} ; U_{i} \mid U_{1}^{i-1}\right)$, it can be seen that the bound, given by $\frac{1}{M} I\left(Y_{1}^{M} ; U_{1}^{M}\right)$, is indeed the biting constraint among the constraints in the Berger-Tung inner bound. In other 
words, by choosing the rates in this way, one can satisfy all of the $\left(2^{M}-1\right)$ constraints given in the Berger-Tung bound. Hence

$$
\begin{aligned}
\frac{1}{M} \sum_{i=1}^{M} R_{i} & =\frac{1}{M} I\left(Y_{1}, \ldots, Y_{M} ; U_{1}, \ldots, U_{M}\right) \\
& =\frac{1}{2 M} \log \prod_{i=1}^{M}\left(\frac{\lambda_{i}}{\phi_{N}}+1\right)
\end{aligned}
$$

where the last equality is one that can also be shown. Hence the following average rate of transmission and average distortion are achievable:

$$
\begin{gathered}
R^{(N, M)}\left(\phi_{N}\right)=\frac{1}{2 M} \sum_{i=1}^{M} \log \left(\frac{\lambda_{i}}{\phi_{N}}+1\right) \\
D^{(N, M)}\left(\phi_{N}\right)=\frac{1}{M} \sum_{i=1}^{M} \frac{\lambda_{i}}{\frac{\lambda_{i}}{\phi_{N}}+1} .
\end{gathered}
$$

Using the Grenander-Szego [7] eigenvalue distribution theorem, as $M \rightarrow \infty$, the following average rate of transmission and average distortions are achievable:

$$
\begin{gathered}
R^{(N, \infty)}\left(\phi_{N}\right)=\frac{1}{4 \pi} \int_{-\pi}^{\pi} \log \left(\frac{\Phi_{N}(w)}{\phi_{N}}+1\right) d w \\
D^{(N, \infty)}\left(\phi_{N}\right)=\frac{1}{2 \pi} \int_{-\pi}^{\pi}\left(\frac{\Phi_{N}(w)}{\frac{\Phi_{N}(w)}{\phi_{N}}+1}\right) d w
\end{gathered}
$$

where $\Phi_{N}(w)$ is the discrete-space power spectral density of the process which is obtained by sampling the original $X^{t}$ process with space-sampling interval $\tau=1 / N$.

Note that now we have a parametric formula, with parameter $0 \leq \phi_{N}<\infty$, for an achievable rate-distortion pair for the discrete-space random process. Since the spacesampling frequency is $N$ sensors/unit distance, the achievable rate in terms of bits per unit distance is given by $N R^{(N, \infty)}$. Further, note that the distortion $D^{(N, \infty)}$ is the mean squared error for the discrete-space process, i.e. for the continuousspace process at the sampling locations. By taking the limit as $N \rightarrow \infty$ we get the following upper-bound on the optimal rate-distortion function of the original continuous-space discrete-time process in the distributed setting.

Theorem An upper bound on the optimal rate-distortion function in the distributed setting is given by the parametric formula: for any $\theta>0$

$$
\begin{gathered}
R(\theta)=\frac{1}{4 \pi} \int_{-\infty}^{\infty} \log \left(\frac{S(\Omega)}{\theta}+1\right) d \Omega \\
D(\theta)=\frac{1}{2 \pi} \int_{-\infty}^{\infty}\left(\frac{S(\Omega)}{\frac{S(\Omega)}{\theta}+1}\right) d \Omega
\end{gathered}
$$

\section{PROOF OF THEOREM}

We follow the techniques used in [10] to represent the achievable rate-distortion pairs in terms of the continuous-space power spectral density. First let us consider the expression for the achievable rate using the Berger-Tung method described in the previous section. As mentioned there, for any $N$ and $\phi_{N}>0$, rate $N R^{N, \infty}\left(\phi_{N}\right)$ bits per unit distance is achievable. With the goal of having this rate approach a finite constant as $N \rightarrow \infty$, we choose $\phi_{N}$ to be proportional to $N$. That is, for some $\theta>0$, we let $\phi_{N}=\theta N$. We now have the following sequence of equalities:

$$
\begin{aligned}
R(\theta) & \stackrel{\Delta}{=} \lim _{N \rightarrow \infty} N R^{(N, \infty)}(N \theta) \\
& =\lim _{N \rightarrow \infty} \frac{N}{4 \pi} \int_{-\pi}^{\pi} \log \left(\frac{\Phi_{N}(w)}{N \theta}+1\right) d w \\
& =\lim _{N \rightarrow \infty} \frac{1}{4 \pi} \int_{-\pi N}^{\pi N} \log \left(\frac{\Phi_{N}\left(\frac{\Omega}{N}\right)}{N \theta}+1\right) d \Omega \\
& =\lim _{N \rightarrow \infty} \frac{1}{4 \pi} \int_{-\infty}^{\infty} I_{N}(\Omega) \log \left(\frac{\Phi_{N}\left(\frac{\Omega}{N}\right)}{N \theta}+1\right) d \Omega \\
& =\frac{1}{4 \pi} \int_{-\infty}^{\infty} \lim _{N \rightarrow \infty} I_{N}(\Omega) \log \left(\frac{\Phi_{N}\left(\frac{\Omega}{N}\right)}{N \theta}+1\right) d \Omega \\
& =\frac{1}{4 \pi} \int_{-\infty}^{\infty} \log \left(\frac{S(\Omega)}{\theta}+1\right) d \Omega
\end{aligned}
$$

where $I_{N}(\Omega)$ equals one if $|\Omega| \leq \pi N$ and zero if not, where the fifth equality uses the bounded convergence theorem [11], while assuming that $\frac{1}{N} \Phi_{N}(\Omega / N)$ is bounded almost everywhere, and where the sixth uses the fact that $\frac{1}{N} \Phi_{N}(\Omega /$ $N)$ converges to $S(\Omega)[10]$.

Next, we consider distortion. Using the method given above, it can be similarly shown that

$$
\begin{aligned}
D^{\prime}(\theta) & \stackrel{\Delta}{=} \lim _{N \rightarrow \infty} D^{(N, \infty)}(N \theta) \\
& =\frac{1}{2 \pi} \int_{-\infty}^{\infty}\left(\frac{S(\Omega)}{\frac{S(\Omega)}{\theta}+1}\right) d \Omega .
\end{aligned}
$$

Note that although $D^{\prime}(\theta)$ is the limit of the MSE in the reconstruction of the discrete-space random process, as mentioned earlier, it can be shown that there exists a sequence (indexed by $N$ ) of methods of reconstructing the original continuous-space random process from the quantized samples of the corresponding discrete-space random process (obtained with sampling frequency $N$ ) such that limit $D(\theta)$ of the MSE for the continuous-space process equals $D^{\prime}(\theta)$. This finishes the proof that $(R(\theta), D(\theta))$ is an achievable rate-distortion pair, and the theorem follows.

\section{EXAMPLE}

Let us consider the case that for each $t, X^{t}(s)$ is a GaussMarkov source with continuous-space power spectral den- 
sity given by $S(\Omega)=2 /\left(1+\Omega^{2}\right)$. Using the following identity that for any $a, b>0$,

$$
\int \log \left(a+b x^{2}\right) d x=x \log \left[\frac{\left(a+b x^{2}\right)}{e^{2}}\right]+\frac{2 \sqrt{a}}{\sqrt{b}} \tan ^{-1}\left(\frac{\sqrt{b} x}{\sqrt{a}}\right)
$$

we can evaluate the upper bound, derived in the previous section, on the optimal rate-distortion function for the distributed setting as given in the following. The parametric form of the rate-distortion function is given by

$$
\begin{gathered}
R(\theta)=\frac{1}{2}\left[\frac{\sqrt{\theta+2}}{\sqrt{\theta}}-1\right] \text { nats, } \\
D(\theta)=\frac{\sqrt{\theta}}{\sqrt{\theta+2}}
\end{gathered}
$$

for $0 \leq \theta<\infty$. Hence the upper bound on the optimal ratedistortion function for the case of Gauss-Markov source is given by

$$
R(D)=\frac{1}{2}\left[\frac{1}{D}-1\right]
$$

It is interesting to note that at $D=0.1$ the upper bound is 4.5 , which is approximately half the value found of the upper bound found in [5]. It is also interesting to compare the distortion profile obtained in this setting with that obtained in optimum centralized source coding (inverse water pouring). Both of these are plotted in Figure 1 for the case when $D=1 / 3$ and $R=1$ bit/unit distance.

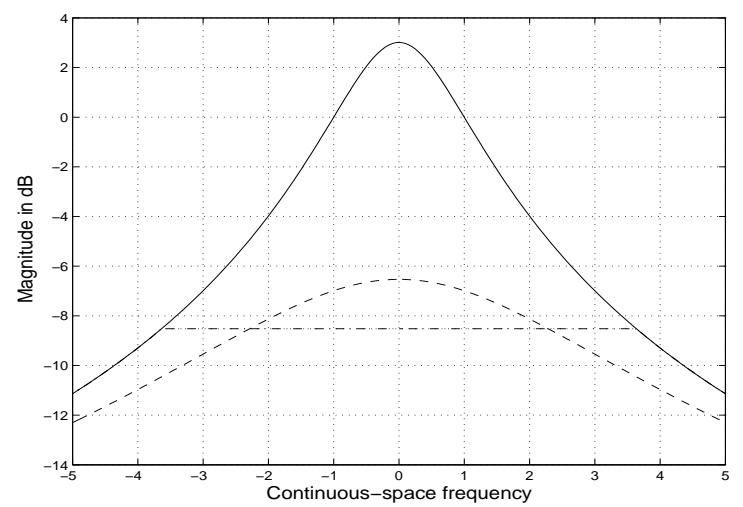

Fig. 1. Gauss-Markov source: The solid curve denotes the continuous-space power spectral density, the dashed curve denotes the distortion profi le obtained in distributed source coding, and the dash-dotted curve denotes the distortion profi le obtained in the optimum centralized source coding (inverse water pouring).

\section{CONCLUSIONS}

By exploiting the Berger-Tung inner bound for distributed coding and the Grenander-Szego asymptotic eigenvalue theory, a new upper bound to the rate of ideal distributed lossy source coding of densely sampled data has been found. The results suggest that this bound is substantially tighter than the previous bound of [5]. On the other hand, the latter bound applies to a fixed finite value of the number of samples $M$, whereas the new bound applies to the limiting case that $M$ is large.

\section{REFERENCES}

[1] A. Scaglione and S. Servetto, "On the interdependence of routing and data compression in multi-hop sensor networks," Proceedings of MOBICOM, 2002.

[2] D. Marco, E. J. Duarte-Melo, M. Liu, and D. L. Neuhoff, "On the many-to-one trnsport capacity of a dense wireless sensor network and the compressibility of its data," Proc. Information processing in sensor networks (IPSN), 2003.

[3] P. Ishwar, A. Kumar, and K. Ramchandran, "Distributed sampling for dense sensor networks: A 'bitconservation principle," Int. Symp. on Inform. Proc. in Sensor Networks (IPSN), Palo Alto, CA, April 2003.

[4] D. Marco and D. L. Neuhoff, "Entropy of quantized data at high sampling rates," Int. Symp. on Information Theory (ISIT), September Adelaide, Australia, 2005.

[5] A. Kashyap, L. A. Lastras-Montano, C. Xia, and Z. Liu, "Distributed source coding in dense sensor networks," Proceedings of the Data Compression Conference (DCC), April, Snowbird, UT, 2005.

[6] T. Berger, "Multiterminal Source Coding," Information Theory Approach to Communication, (CISM Courses and Lecture Notes No. 229), G. Longo, Ed., Wien and New York: Springer-Verlag, 1977.

[7] U. Grenander and G. Szego, Toeplitz forms and their applications. Berkeley, CA: University of California Press, 1958.

[8] A. B. Wagner, S. Tavildar, and P. Viswanath, "The rate region of the quadratic Gaussian two-terminal sourcecoding problem," www.arxiv.org/abs/cs.IT/0510095.

[9] A. D. Wyner, "The rate-distortion function for source coding with side information at the decoder-II: General sources," Inform. Contr., vol. 38, pp. 60-80, 1978.

[10] T. Berger, Rate distortion theory: A mathematical basis for data compression. Englewood Cliffs: Prentice Hall, 1971.

[11] H. L. Royden, Real analysis. Englewood Cliffs: Prentice Hall, 1988. 